\title{
A proteinúria como fator de risco para retinopatia diabética
}

\author{
Proteinuria as a risk factor for diabetic retinopathy
}

\author{
André Moraes Freitas ${ }^{1}$ \\ Zélia Maria da S. Corrêa ${ }^{2}$ \\ Ítalo Mundialino Marcon ${ }^{3}$ \\ Helena Schmidt ${ }^{4}$
}

\begin{tabular}{l} 
RESUMO \\
\hline Introdução: A diabete mélito é doença metabólica complexa que envolve \\
hiperglicemia, doença microvascular(retina erim) eneuropatia. Aretinopatia \\
e a nefropatia são importantes causas de cegueira e falência renal respec- \\
tivamente, e complicações relacionadas à diabete mélito. Objetivo: Deter- \\
minar a relação entre a presença de proteinúria e nefropatia coma gravidade \\
da retinopatia diabética num estudo transversal de pacientes diabéticos. \\
Métodos: Estudo transversal de pacientes diabéticos, sem tratamento \\
oftalmológico prévio, atendidos em serviço de oftalmologia terciário. Estes \\
pacientes foram submetidos a exame fundoscópico, exames laboratoriais \\
e interrogados quanto ao tempo de duração e tipo de diabete. Compara- \\
dos os fatores de risco abordados com os achados fundoscópicos. Na \\
análise dos dados quantitativos foi usado o teste t de Student. Resultados: \\
Estudados 81 pacientes, 28 do sexo masculino, 53 do sexo feminino, 28 com \\
diabete insulino-dependente 53 com diabete não-insulino-dependente. \\
Fatores correlacionados estatisticamente com o grupo com retinopatia \\
diabética mais grave incluem: diabete mélito insulino dependente $(\alpha<0,01)$, \\
nefropatia( $\alpha<0,05)$, proteinúria( $\alpha<0,05)$ maior tempo de doença(p<0,001) \\
e valores mais elevados de glicemia de jejum (p=0,01). Conclusões: \\
Concluiu-se que a gravidade de retinopatia diabética está relacionada à \\
presença de proteinúria e nefropatia além de sofrer influência de fatores de \\
risco tais como tempo de duração da doença, tipo de diabete e controle \\
metabólico da doença.
\end{tabular}

Descritores: Diabetes mellitus; Retinopatia diabética; Proteinúria; Fatores de risco; Nefropatias

\footnotetext{
${ }^{1}$ Residente do Serviço de Oftalmologia da Sta. Casa de Porto Alegre/ Fundação Faculdade Federal de Ciências Médicas de Porto Alegre (FFFCMPA) - RS

${ }^{2}$ Responsável pelo Setor de Retina da Santa Casa de Porto Alegre - Porto Alegre - RS. Pós-Graduanda, Nível Doutorado, UNIFESP (Universidade Federal de São Paulo) - SP.

${ }^{3}$ Chefe do Serviço de Oftalmologia da Santa Casa de Porto Alegre - Porto Alegre - RS. Doutor em Oftalmologia e Professor Regente da Disciplina de Oftalmologia da FFFCMPA, Porto Alegre - RS.

${ }^{4}$ Chefe do Serviço de Endocrinologia da Santa Casa Porto Alegre - RS. Mestra e Doutora em Medicina. Professora Titular da Disciplina de Endocrinologia da FFFCMPA, Porto Alegre - RS

Endereço para correspondência: Av. Nilo Peçanha, 2421 - Porto Alegre (RS). CEP 91330-001.

E-mail:zmcorrea@terra.com.br

Recebido para publicação em 08.01.2001

Aceito para publicação em 20.08.2001
}

\section{INTRODUÇÃO}

A diabete mélito é uma doença metabólica complexa, resultante da interação variável entre fatores hereditários e ambientais. Caracteriza-se por secreção anormal de insulina, níveis elevados de glicose sangüínea e uma variedade de complicações em órgãos essenciais para a manutenção da vida, incluindo nefropatia, retinopatia, neuropatia e aterosclerose acelerada. A síndrome clínica completa envolve hiperglicemia, doença microvascu$\operatorname{lar}$ (retina e rim) e neuropatia ${ }^{(1)}$. A retinopatia e a nefropatia são importantes causas de cegueira e falência renal respectivamente, e complicações relacionadas à diabete mélito ${ }^{(2)}$.

A retinopatia diabética é a principal causa de cegueira em americanos com idade entre 20 e 74 anos, sendo responsável por $12 \%$ de todos os casos de novos cegos em um ano ${ }^{(3-4)}$. A prevalência da retinopatia diabética apresenta uma variação muito grande segundo a literatura, dependendo basicamente da população em estudo, variando de $18 \%$ a $40 \%{ }^{(5)}$. Calcula-se que 1 a $3 \%$ da população mundial esteja acometida pela doença. Aproxima- 
damente $85 \%$ dos casos se manifestam após os 40 anos de idade, sendo apenas 5\% abaixo dos 20 anos. No Brasil, as estatísticas são insuficientes. Trabalhos publicados no VII Congresso de Prevenção da Cegueira mostraram uma prevalência de olhos cegos variando de 1,42 a 9,77\% devido à retinopatia diabética ${ }^{(6)}$.

As alterações do fundo de olho do paciente diabético seguem um curso progressivo, partindo de retinopatias leves, não proliferativas, cuja manifestação precoce é o desenvolvimento de microaneurismas ${ }^{(7)}$, para as graves, proliferativas, quando se evidencia a formação de neovasos ${ }^{(3-4)}$.

As complicações retinianas dependem não apenas do deficiente metabolismo de carboidratos como também de outros fatores sendo pesquisados atualmente ${ }^{(7)}$. Há uma série de fatores relacionados ao desenvolvimento e progressão da retinopatia diabética, alguns mais fortemente associados com a gravidade da retinopatia, outros menos. Não é possível definir quais indivíduos diabéticos apresentarão retinopatia, entretanto, é possível definir fatores de risco para o desenvolvimento da doença ${ }^{(6)}$ como: duração da doença sistêmica, mau controle metabólico, diabete melito insulino dependente, doença renal associada, etc ${ }^{(1-2,7-12)}$. Alguns estudos sugerem que a velocidade de progressão da retinopatia é menor em indivíduos diabéticos jovens (menores de 13 anos de idade). Acredita-se que as causas relacionam-se com as alterações hormonais da puberdade ${ }^{(6)}$.

Estudos epidemiológicos acerca da incidência, prevalência e progressão da retinopatia diabética são importantes para estabelecer hipóteses da sua patogênese e para desenvolver estratégias de prevenção desta manifestação da doença. Estes dados possibilitam o aconselhamento médico adequado, embasado em evidências. Estes estudos tornam-se ainda mais importantes por evidenciarem que os principais fatores de risco são evitáveis ou, ao menos, passíveis de controle.

Este estudo tem por objetivo determinar a relação entre proteinúria e nefropatia com a ocorrência e severidade da retinopatia diabética num estudo transversal de pacientes diabéticos atendidos em ambulatório de oftalmologia de serviço terciário.

\section{MÉTODOS}

Os critérios de exclusão para este estudo foram terapia com fotocoagulação a laser prévia ou cirurgia vítreo-retiniana, idade menor que 13 anos, impossibilidade de realização de exame fundoscópico (pacientes com opacidades de meios) e a presença de hipertensão arterial descompensada.

Todos os pacientes foram submetidos à medida da acuidade visual (com correção ou com uso do buraco estenopeico) usando a tabela do ETDRS (Early Treatment for Diabetic Retinopathy Study) conforme suas recomendações ${ }^{(13)}$ e, em seguida examinados pelo mesmo oftalmologista (ZMSC), com oftalmoscópio indireto e lente condensadora de 20 dioptrias, após dilatação pupilar com colírio midriático (tropicamida 1\%).
Foram realizados os seguintes exames laboratoriais: glicemia de jejum, hemoglobina glicosilada, dosagem da creatinina plasmática e exame qualitativo de urina. Foi definido como nefropatia a presença de proteinúria ao exame quantitativo de urina e/ou creatinina plasmática $>1,3 \mathrm{mg} / \mathrm{dl}$.

Os pacientes foram interrogados quanto ao tipo de diabete (insulino-dependente e não insulino-dependente) e duração da doença desde o diagnóstico.

Os pacientes foram classificados em quatro grupos, segundo estágios crescentes de severidade de retinopatia diabética, baseados no Early Treatment Diabetic Retinopaty Research Group $^{(8)}$, da seguinte forma:

- Grupo 1: retinopatia diabética não proliferativa muito leve a leve, nos quais observam-se apenas microaneurismas e/ou hemorragias intra-retinianas e exsudatos duros. Foram incluídos neste grupo os pacientes diabéticos com exame fundoscópico sem alterações.

- Grupo 2: retinopatia não proliferativa moderada, incluindo a presença de microaneurismas e/ou hemorragias intraretinianas leves a moderadas em quatro quadrantes ou severos em menos de quatro quadrantes, exsudatos duros e algodonosos.

- Grupo 3: retinopatia não proliferativa severa a muito severa, observando-se características de risco para proliferação, como alterações microvasculares intra-retinianas (IRMAs), veias em rosário e áreas isquêmicas extensas.

- Grupo 4: retinopatia proliferativa, evidenciando-se a presença de neovasos e/ou proliferações vítreo-retinianas.

Após a coleta de dados, foi investigada a possível associação entre proteinúria, nefropatia e a presença e severidade da doença retiniana revelada ao exame fundoscópico.

Devido ao fato deste ser um estudo transversal em indivíduos sem tratamento ocular prévio, a maioria dos pacientes apresentavam estágios iniciais da retinopatia. Para efeito de cálculo estatístico dos dados, os pacientes do grupo 1 foram chamados: grupo A, e os pacientes dos grupos 2, 3 e 4, agrupados no grupo B. Tal separação favorece a análise dos dados ao separar pacientes sem alterações ou com retinopatia inicial dos pacientes com retinopatia mais avançada, não comprometendo a validade do estudo e permitindo o cálculo do risco relativo de variáveis qualitativas. Assim, temos que o grupo A corresponde aos pacientes sem retinopatia ou com retinopatia leve, e o grupo B corresponde aos pacientes com retinopatia moderada a proliferativa. A distribuição dos pacientes de acordo com os dois grupos descritos é apresentada na tabela 3. Foi realizada a análise estatística comparando estes dois grupos com os fatores de risco em estudo.

\section{RESULTADOS}

Foram selecionados 81 pacientes diabéticos atendidos em ambulatório de oftalmologia de referência que não apresentavam os critérios de exclusão. A idade dos pacientes variou de

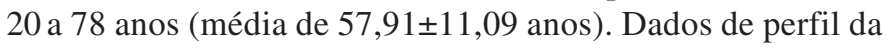


amostra como sexo e tipo de diabete são expostos nas tabelas 1 e 2 respectivamente.

A distribuição dos pacientes segundo a classificação da retinopatia nos quatro grupos descritos anteriormente foi realizada considerando-se o olho com doença mais avançada. Os resultados são apresentados na tabela 3 .

A acuidade visual do grupo está apresentada no gráfico 1. Considerando os 81 pacientes estudados, somente 2 olhos não apresentavam percepção luminosa (1 olho phithísico devido a trauma ocular e outro por glaucoma neovascular e complicações relacionadas à retinopatia diabética). Em 158 olhos foi acessado o status macular (sendo que 4 olhos foram excluídos: 2 por razões acima descritas e outros 2 por opacidades dos meios (catarata)). Destes, 21 olhos apresentavam alguma alteração macular como edema clinicamente significativo, espessamento e/ou depósito lipídico em uma área de 500 micra em torno da fóvea. Em quase todos os olhos com alteração macular (exceto 2) a acuidade visual se apresentou pior que $0,2(n=19)$.

Doze pacientes do grupo A $(n=54)$ e 15 pacientes do grupo B $(n=27)$ apresentaram proteinúria ao exame qualitativo de urina. Entretanto, somente 1 paciente do grupo A e 2 pacien-

\begin{tabular}{|lc|}
\hline & Tabela 1. Distribuição por sexo \\
Sexo & Número de pacientes \\
Masculino & $28(35 \%)$ \\
Feminino & $53(65 \%)$ \\
Total & $81(100 \%)$ \\
\hline
\end{tabular}

Tabela 2. Distribuição por tipo de diabete

\section{Tipo de diabete}

Insulino dependente

Não insulino dependente

Total
Número de pacientes

$28(35 \%)$

$53(55 \%)$

$81(100 \%)$
Tabela 3. Distribuição dos pacientes nos grupos A e B

\begin{tabular}{|lc|} 
Grupo & Número de pacientes \\
A & $54(66,7 \%)$ \\
B & $27(33,3 \%)$ \\
Total & $81(100 \%)$ \\
\hline
\end{tabular}

\begin{tabular}{|lc|}
\hline & Tabela 4. Distribuição nos grupos de retinopatia \\
Grupo & Número de pacientes \\
1 & $54(66,7 \%)$ \\
2 & $10(12,3 \%)$ \\
3 & $10(12,3 \%)$ \\
4 & $7(8,6 \%)$ \\
Total & $81(100 \%)$ \\
\hline
\end{tabular}

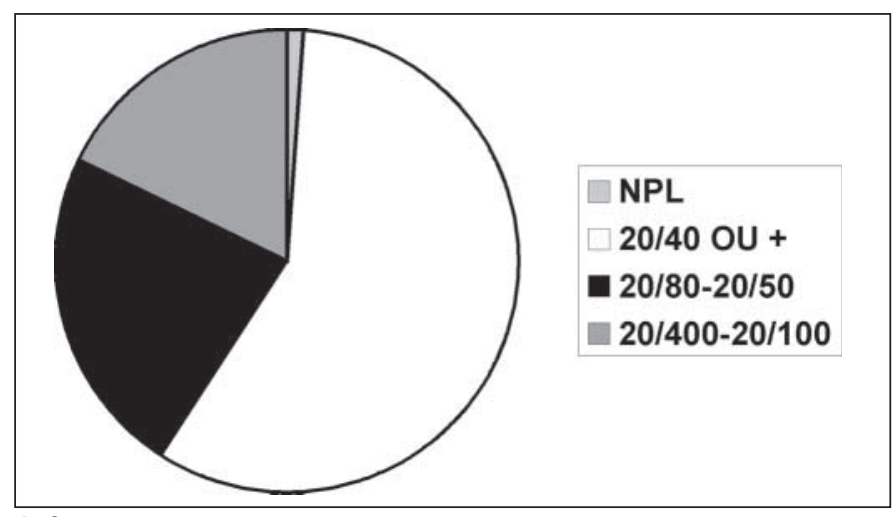

Gráfico 1 - Acuidade visual corrigida ou com uso do buraco estenopeico de 81 pacientes (162 olhos) medida com a tabela do ETDRS (Early Treatment for Diabetic Retinopathy Study)

tes do grupo B apresentaram creatinina superior a $1,3 \mathrm{mg} / \mathrm{dl}$. A nefropatia (definida pela presença de proteinúria e/ou valor da creatinina plasmática acima de $1,3 \mathrm{mg} / \mathrm{dl}$ ) ocorreu em 12 pacientes do grupo A e 15 pacientes do grupo B. Todos os pacientes que apresentaram creatinina aumentada também apresentaram proteinúria.

A análise das variáveis qualitativas foi realizada utilizando-se o teste do Qui-quadrado. Consideramos como estatisticamente significativo $\alpha<0,05$. Desta forma foram analisados os seguintes dados: sexo, tipo de diabete, presença de proteinúria e nefropatia. Encontramos associação de maior gravidade da retinopatia (grupo B) com as seguintes variáveis: diabete melito insulino dependente $(\alpha<0,01)$, presença de proteinúria $(<0,05)$ e nefropatia $(\alpha<0,05)$. O gênero dos pacientes não apresentou correlação estatística com a gravidade de retinopatia diabética $(\alpha>0,05)$.

$\mathrm{Na}$ análise dos dados quantitativos (idade, tempo de doença, glicemia de jejum, hemoglobina glicosilada, creatinina plasmática e acuidade visual) foi usado o teste $\mathrm{T}$ de Student, comparando os Grupos A e B. Foi considerado estatisticamente significativo $\mathrm{p}<0,02$. A maior gravidade de retinopatia diabética (Grupo B) apresentou correlação com maior tempo de doença $(p<0,001)$, valores elevados de glicemia de jejum $(p=0,01)$ e hemoglobina glicosilada elevada $(p=0,001)$. A acuidade visual de pacientes do Grupo B era consideravelmente menor do que pacientes do Grupo A $(\mathrm{p}<0,001)$.

Não houve correlação estatística entre a gravidade de retinopatia e os seguintes dados: idade $(\mathrm{p}=0,88)$ e creatinina plasmática $(\mathrm{p}=0,48)$.

\section{DISCUSSÃO}

Muitos estudos têm examinado os fatores de risco para desenvolvimento e progressão de retinopatia diabética. A duração da diabete parece ser o fator mais fortemente associado com a retinopatia diabética, confirmado por vários estudos ${ }^{(5,9,14-15)}$.

O Diabetes Clinical and Complications Trial (DCCT) ${ }^{(1)}$ 
avaliou os efeitos do controle rigoroso da glicemia e concluiu, em 1993, que em diabéticos juvenis, o tratamento intensivo com insulina retarda o aparecimento e progressão da retinopatia, nefropatia e neuropatia. Outros estudos confirmam tal afirmação, tanto para pacientes com diabete melito tipo I quanto para indivíduos com diabete melito tipo $\mathrm{II}^{(8,16)}$.

Estudos demonstram maior prevalência de retinopatia proliferativa na diabete mélito insulino dependente do que na diabete mélito não insulino dependente ${ }^{(5)}$. Entretanto não foi possível definir se tal associação se deve a diferenças metabólicas dos dois tipos da doença, ou a diferenças nas idades dos pacientes e níveis glicêmicos geralmente maiores em pacientes com diabete melito tipo $\mathrm{I}^{(5)}$.

Cruickshanks avaliou 1139 diabéticos, encontrando associação entre microalbuminúria e a presença de retinopatia nestes pacientes, sugerindo uma influência de nefropatia na doença ${ }^{(13)}$.

Klein e colaboradores estudaram 2980 diabéticos e concluíram que independente de haver relação etiológica entre a retinopatia diabética proliferativa e doença renal, a presença destas é de grande relevância clínica ${ }^{(2)}$. Além disto, seus resultados demonstraram que a proteinúria é fator de risco para o desenvolvimento de retinopatia diabética proliferativa especialmente em indivíduos cujo aparecimento da diabete foi mais precoce $^{(2)}$. Esse estudo também demonstrou a importância da proteinúria como fator de risco, entretanto esta correlação apresentou significância estatística independente da idade do paciente ou do tipo de diabete.

Fatores como dosagem de creatinina plasmática, idade e sexo parecem não exercer qualquer influência na progressão da retinopatia diabética. Os dados aqui obtidos demonstram que a proteinúria como indicador da nefropatia é relevante fator de risco. Entretanto, a creatinina plasmática não se mostrou um bom indicador da nefropatia como fator de risco para o desenvolvimento e severidade da retinopatia diabética.

Outros resultados obtidos neste estudo assemelham-se a maioria dos estudos descritos anteriormente acerca dos fatores de risco para maior gravidade de retinopatia diabética ${ }^{(17)}$. Tempo de doença, diabete mélito insulino dependente e mau controle metabólico da doença são indubitavelmente fatores de grande importância ${ }^{(7,9-11,16-18)}$.

A acuidade visual sofre influência direta da gravidade de retinopatia, sendo prejudicada nos indivíduos com doença mais avançada.

Por ser um estudo transversal, não existe seguimento destes pacientes. Entretanto considerando os dados obtidos, os autores puderam correlacioná-los com a presença e a severidade da retinopatia diabética nos pacientes estudados naquele dado momento.

Concluímos que a nefropatia, determinada pela presença de proteinúria, na população estudada, apresenta correlação com a ocorrência e severidade da retinopatia diabética. Outros fatores que pareceram influenciar a gravidade de retinopatia diabética foram: tempo de duração da doença, tipo de diabete e controle metabólico da doença. Fatores que não pareceram exercer influência incluem idade, sexo e dosagem da creatinina plasmática. Tais dados estão de acordo com a maioria dos estudos anteriormente descritos.

Os autores sugerem que o exame quantitativo de urina, relativamente simples e de baixo custo, seja incluído na avaliação de indivíduos com retinopatia diabética uma vez que pode auxiliar sua avaliação sistêmica e no planejamento do acompanhamento clínico oftalmológico.

\section{ABSTRACT}

Introduction: Diabetes mellitus is a complex metabolic disease characterized by abnormal insulin secretion, elevated fasting plasma glucose, and a variety of complications in terminal organs. Retinopathy and nephropathy are important causes of blindness and renal failure respectively, and complications related to diabetes mellitus. Purpose: To determine the relationship between the presence of proteinuria and nephropathy and the severity of diabetic retinopathy. Methods: Transversal study of diabetic patients without previous ophthalmologic treatment, seeing at a reference eye clinic. These patients underwent indirect ophthalmoscopy and blood work- up, and were questioned about duration and type of diabetes mellitus. The presence of risk factors were correlated with the fundus findings. The statistical analysis of the quantitative data was performed using Student's t test. Results: Eightyone patients were selected, 28 male, 53 female, 28 had insulindependent diabetes mellitus, 53 had non-insulin-dependent diabetes mellitus. Factors statistically related to more severe diabetic retinopathy include: insulin-dependent diabetes mellitus $(\alpha<0.01)$, nephropathy $(\alpha<0.05)$, proteinuria $(\alpha<0.05)$, duration of the disease $(\mathrm{p}<0.001)$, and elevated fasting plasma glucose $(\mathrm{p}=0.01)$. Conclusions: The severity of diabetic retinopathy appears to be associated with the presence of proteinuria and nephropathy besides other risk factors such as duration of disease, type of diabetes, and poor metabolic control. The authors suggest that a quantitative urine test should be included in the evaluation of diabetic retinopathy.

Keywords: Diabetes mellitus; Retinopathy/diabetic; Proteinuria; Risk factors; Kidney diseases

\section{REFERÊNCIAS}

1. DCCT Research Group. The effect of intensive treatment of diabetes on the development and progression of long-term complications in insulin-dependent diabetes mellitus. N Engl J Med 1993;329:977-86.

2. Klein R, Moss SE, Klein BEK. Is gross proteinuria a risk factor for the incidence of proliferative diabetic retinopathy? Ophthalmology 1993;100:1140-6.

3. Olk RJ, Lee CM. Diabetic retinopathy: practical management. Philadelphia, London: Lippincott; 1993. p.1-39.

4. Dorchy H. Characterization of early stages of diabetic retinopathy. Importance of the breakdown of the blood retinal barrier. Diabetes Care 1993;16:1212-3.

5. Kanski J. Diabetic Retinopathy. In: Kanski J. Clinical ophthalmology. London: Butterworth-Heinemann; 1994. p.344-57. 
6. Souza EC, Esteves JF, Broilo VR, Domingues CG, Lavinsky J. Retinopatia diabética não proliferativa. In: Abujamra S, Ávila M, Barsante C, Farah ME, Gonçalves JOR, Lavinsky J et al. editores. Retina e vítreo: Clínica e cirurgia. São Paulo: Rocca; 2000. p.485-99.

7. Kohner EM, Aldington SJ, Stratton IM, Manley SE, Holman RR, Matthews $\mathrm{DR}$, et al. Diabetic retinopathy at diagnosis of non-insulin-dependent diabetes mellitus and associated risk factors. Arch Ophthalmol 1998:116:297-303.

8. Araki A, Ito H, Hattori A, Inoue J, Sato T, Shiraki M, Orimo H. Risk factors for development of retinopathy in elderly Japanese patients with diabetes mellitus. Diabetes Care 1993;16:1184-6.

9. Molyneaux LM, Constantino MI, McGill M, Zilkens R, Yue DK. Better glycaemic control and risk reduction of diabetic complications in type 2 diabetes: comparison with DCCT. Diabetes Res Clin Pract 1998;42:77-83.

10. Okada S, Ichiki K, Tanokuchi S, Hamada H, Matsuo N, Ota Z. Factors related to the development and progression of diabetic retinopathy in patients with type 2 diabetes. J Int Med Res 1996;24:214-20.

11. Cruickshanks KJ, Ritter LL, Klein R, Moss SE. The association of microalbuminuria with diabetic retinopathy. The Wisconsin epidemiologic study of diabetic retinopathy. Ophthalmology 1993;100:862-7.
12. Yoshizumi MO. The medical control of diabetic retinopathy. In: Lewis $\mathrm{H}$, Ryan SJ editors. Medical and surgical retina. London: Mosby;1994. p. 257-68.

13. Early Treatment Diabetic Retinopathy Study Research Group - ETDRS report number 7. Early treatment diabetic retinopathy study design and baseline patient characteristics. Ophthalmology 1991;98(5 Suppl):741-56.

14. McCance DR, Hadden DR, Atkinson AB, Archer DB, Kennedy L. Long-term glycaemic control and diabetic retinopathy. Lancet 1989;2:824-8.

15. Guillausseau PJ, Massim P, Charles MA, Allaguy H, Guvenli Z, Virally M, Tielmans D, Assayag M, Warnet A, Lubetzki J. Glycaemic control and development of retinopathy in type 2 diabetes mellitus: a longitudinal study. Diabet Med 1998;5:151-5.

16. Frank R N. Etiologic mechanisms in diabetic retinopathy. In: Ryan SJ, editor. Retina. Philadelphia: Mosby; 1994. p.1243-76.

17. Steck AD, Esteves RF, Gonçalves JCM. Prevalência de retinopatia diabética em uma população de diabéticos do $\mathrm{HC}$ de Franco da Rocha. Arq Bras Oftalmol 1993;56:125-8

18. Klein R, Klein BE, Moss SE, Cruickshanks KJ. The Wisconsin epidemiologic study of diabetic retinopathy. XIV. Ten-year incidence and progression of diabetic retinopathy. Arch Ophthalmol 1994;112:1217-28.

\title{
$X X^{\text {th }}$ INTERNATIONAL CONGRESS OF OPHTHALMOLOGY
}

\author{
21 a 25 de Abril de 2002 \\ Sidney - Austrália
}

\section{INFORMAÇÕES: www.ophthalmology.aust.com}

\section{Agência de Turismo Oficial-Brasil:}

\author{
Mello Faro Turismo
}

Fone: (1 1 ) 3258-5044 - Fax: (1 1 ) 3231-1343

E-mail: eventos@mellofaro.com.br 